LAWRENCE LIVERMORE N A T IO N A L LABORATORY
A Report on the use of Weak-Shock Wave Profiles and 3-D Dislocation Dynamics Simulations for Validation of Dislocation Multiplication and Mobility in the Phonon Drag Regime

D.H. Lassila, J.U. Cazamias, M. Shehadeh, H. Zbib

February 24, 2004 
This document was prepared as an account of work sponsored by an agency of the United States Government. Neither the United States Government nor the University of California nor any of their employees, makes any warranty, express or implied, or assumes any legal liability or responsibility for the accuracy, completeness, or usefulness of any information, apparatus, product, or process disclosed, or represents that its use would not infringe privately owned rights. Reference herein to any specific commercial product, process, or service by trade name, trademark, manufacturer, or otherwise, does not necessarily constitute or imply its endorsement, recommendation, or favoring by the United States Government or the University of California. The views and opinions of authors expressed herein do not necessarily state or reflect those of the United States Government or the University of California, and shall not be used for advertising or product endorsement purposes.

This work was performed under the auspices of the U.S. Department of Energy by University of California, Lawrence Livermore National Laboratory under Contract W-7405-Eng-48. 


\title{
A Report on the use of Weak-Shock Wave Profiles and 3-D Dislocation Dynamics Simulations for Validation of Dislocation Multiplication and Mobility in the Phonon Drag Regime
}

\author{
David H. Lassila, James U. Cazamias, \\ Lawrrence Li vermore National Laboratory, Livermore, CA 94551
}

Mu'tasem Shehadeh and Hussein Zbib

School of mechanical and Materials Engineering, Washington State University, Pullman, WA 99163-2920, USA

February 19, 2004

\begin{abstract}
Dynamically loaded gas gun experiments were performed to validate the predictive capabilities of 3-D dislocation dynamics (DD) code simulations at very high strain rates and dislocation velocities where the phonon drag mechanism will be dominant. Experiments were performed in the weak-shock regime on high-purity Mo single crystals with [001] compression axes. We have also performed shock-recovery experiments and are in the process of analyzing the dislocation structure generated by the weak-shock using transmission electron microscopy (TEM), which will also be used to validate the dislocation structure predicted by the DD simulations. The DD simulations being performed at Washington State University by Prof. H. Zbib and co-workers will be compared to the experimentally measured wave profiles, thereby validating mechanisms of dislocation generation and motion. Some DD simulation results are presented to demonstrate the feasibility of using a combined experimental/simulation effort for the validation of dislocation generation and mobility physics issues in the phonon drag regime.
\end{abstract}

\subsection{Introduction; weak-shock wave profiles for strength measurements}

In the weak shock regime, where strain rates are typical on the order of $10^{4}-$ $10^{6} \mathrm{~s}^{-1}$, the initial planar shock wave decomposes into two waves - a strong elastic wave traveling at the longitudinal sound speed and a plastic wave, which travels at a slower speed. The magnitude, shape, and decay rate of the wave form gives a measure of the dynamic yield point of the material and work hardening behavior related to dislocation generation and motion. Planar impact experiments, as illustrated in figure 1 are a welt established means of accessing these high strain rate conditions in materials. When the experimental data is combined with modeling which accounts for the high-pressure equation of state (EOS), strength parameters such as yield strength, work hardening and viscoplasticity can be quantified [for example see Steinberg, 1989]. 
In bcc metals the plastic strain rate associated with weak-shocks are generally believed to correspond to dislocation velocities in the "phonon drag" regime. This conclusion is primarily based on the observation that the dynamic yield strength, commonly referred to as the Hugoniot elastic limit (HEL), is found to be substantially greater than experimental estimates of the Peierls stress. This makes the weak-shock wave-profile experiment ideally suited for studies of dislocation behavior in the phonon drag regime.

Due to the dynamic nature of the weak-shock experiments, modeling efforts that take into account the EOS of the study material must be coupled with the experimental results to determine strength properties. In the past, the majority of these efforts were essentially 1$\mathrm{D}$ in nature and employed isotropic elastic properties and isotropic $\mathbf{J}_{2}$ plasticity models. These modeling efforts are not capable of accounting for the details associated with crystal plasticity and dislocation activity which are fundamental to the plasticity that occurs in polycrystalline metals. While atomistic simulation are now capable of simulation the passage of strong shock with very small rise times (strain rates greater than $10^{7} \mathrm{~s}^{-1}$ ), they can not be employed to model the propagation of weak-shocks because the shock event is orders of magnitude greater than the time span that can be simulated.

Recently developed 3-D dislocation dynamics (DD) simulations are in many ways ideally suited for simulation of the propagation of weak-shocks. Current DD simulations can account for: 1) all of the fundamental dislocation physics issues associated with crystal plasticity, 2) plasticity events that occur of a time span on the order of $10 \mathrm{~ms}$ and 3) large enough volumes of material to simulated the entire shock rise, shock dwell, and shock release associated with weak-shocks.

In this work we carry out a series of planar weak-shock experiments on single crystal samples of Ta with a [100] orientation with respect to the shock propagation. Also, shock / soft-recovery experiments were performed on identical Ta single crystal samples to allow examination of the microstructural changes that have taken place due to shock loading. Preliminary 3-D DD simulations of shock propagation in $\mathrm{Cu}$ single crystals are presented to demonstrate the feasibility of using a combined experimental / DDsimulation approach to study the behavior of dislocation in the phonon-drag regime.

\section{Experimental}

\subsection{Wave Profiles}

The experiments were performed on high-purity Ta single crystals oriented such that the propagation of the weak-shock was in the [001] crystallographic direction. The Ta sample was backed by a sapphire window, and the shock loading was via a aluminum flyer plate. The sapphire was coated with gold and the Ta was attached to the gold surface with an adhesive. The sapphire remains elastic during the experiment and is a relatively good impedance match with $\mathrm{Ta}$, and hence allowed a measurement of the particle velocity at the $\mathrm{Ta}$ /sapphire interface with minimal perturbation of the waveform. 
Since the goal of this work is to be able to have a direct comparison between experimental data and simulation results, the sample thickness was chosen so that the entire experiment could be simulated using 3-D DD codes, which are currently limited to simulation volumes that are on the order of $10^{3}-10^{4} \mu \mathrm{m}^{3}$ (with typical dislocation densities on the order of $10^{9}-10^{16} \mathrm{~m}^{-1}$ ). The thickness of our test samples were chosen to be $500 \mu \mathrm{m}$ thick. This thickness is very thin compared to the usual thickness of targets used for shock experiments such as these, but allows the full thickness of the sample to be simulated using 3-D dislocation dynamics with a reasonable cross sectional area (on the order of $20 \mu \mathrm{m}^{2}$ ).

The particle velocity was measured at the Ta-sapphire interface using a VISAR (fringe constant of $60 \mathrm{~m} / \mathrm{s}$ ). Ten experiments were performed. Two were outright failures (Shots 1052 and 1053). Of the remaining eight, six (Shots 1134, 1281, 1283, 1284, 1285, 1286) suffered a lost of contrast in the elastic portion of the wave, although they are consistent with the two successful shots (Shots 1133 and 1282) in that total rise times and the shapes of the plastic portion of the wave are similar. While heterogeneous behavior cannot be completely ruled out, we believe that the difference is due to being on the edge of the detectors time response. To get around this, a VISAR with a streak camera as a detector should be used. An open beam VISAR would also provide additional temporal resolution.

Laying the profiles on top of each other via temporal translation (not scaling), the profiles exhibit differences at the base of the wave, the peak of the wave and the elastic-plastic transition. The $5 \mathrm{~m} / \mathrm{s}$ feature at the base of Shot 1133 is an experimental artifact and should be ignored. The difference at the peak of the wave is due to the faster impact velocity of Shot 1282 resulting in a larger peak stress with a corresponding higher plastic wave speed. The difference at the elastic-plastic transition is not easily explainable. Shot 1133 exhibits a higher yield stress and "viscoplastic" behavior. Shot 1282 exhibits a lower yield and a plastic shock followed by "viscoplastic" behavior. Analysis of the recovered samples will provide insight into this discrepancy. One should note that the "viscoplastic" behavior for both experiments is very similar, that is, the shape of the wave between the HEL and the top of the plastic wave.

Table 1. Shot parameters

\begin{tabular}{|l|l|l|}
\hline & Shot 1133 & Shot 1282 \\
\hline Target & {$[100] \mathrm{Ta}$} & {$[100] \mathrm{Ta}$} \\
\hline Thickness ( m) & 497 & 486 \\
\hline Base Plate & C-cut sapphire & R-cut sapphire \\
\hline Impactor & $\mathrm{Al}$ & $\mathrm{Al}$ \\
\hline Thickness $(\mathrm{mm})$ & 1.476 & 1.490 \\
\hline Impact Velocity (m/s) & 490 & 520 \\
\hline Nominal Peak Stress (kbar) & 62 & 66 \\
\hline Glue Layer Thickness ( m) & $<10$ & $<1$ \\
\hline
\end{tabular}




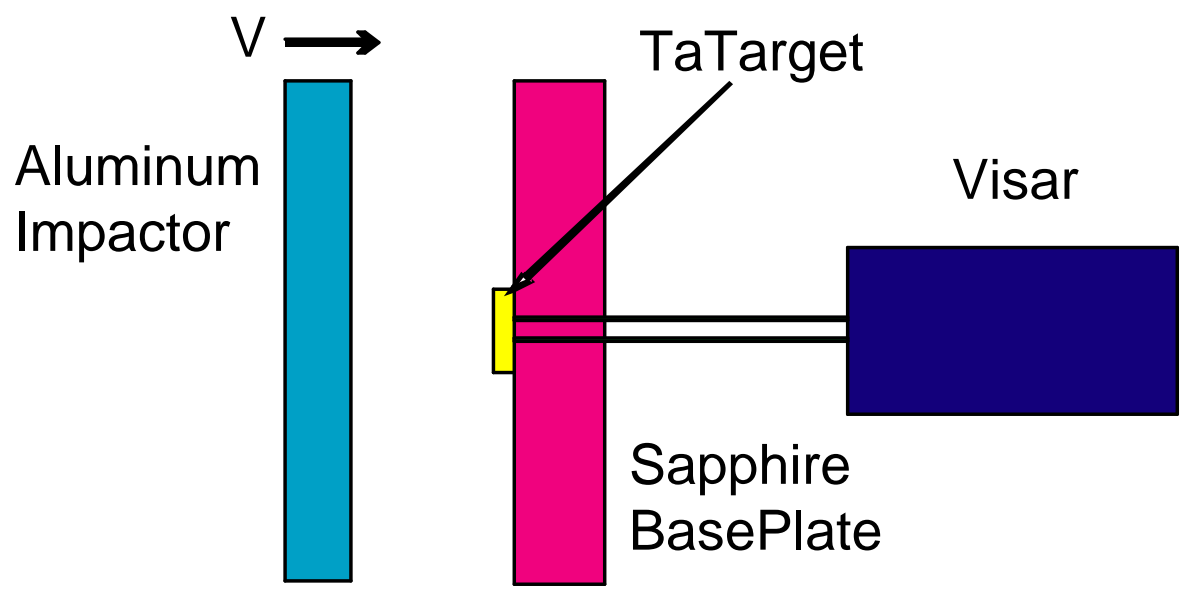

Figure 1. Schematic of the single crystal wear-shock wave-profile experiments.

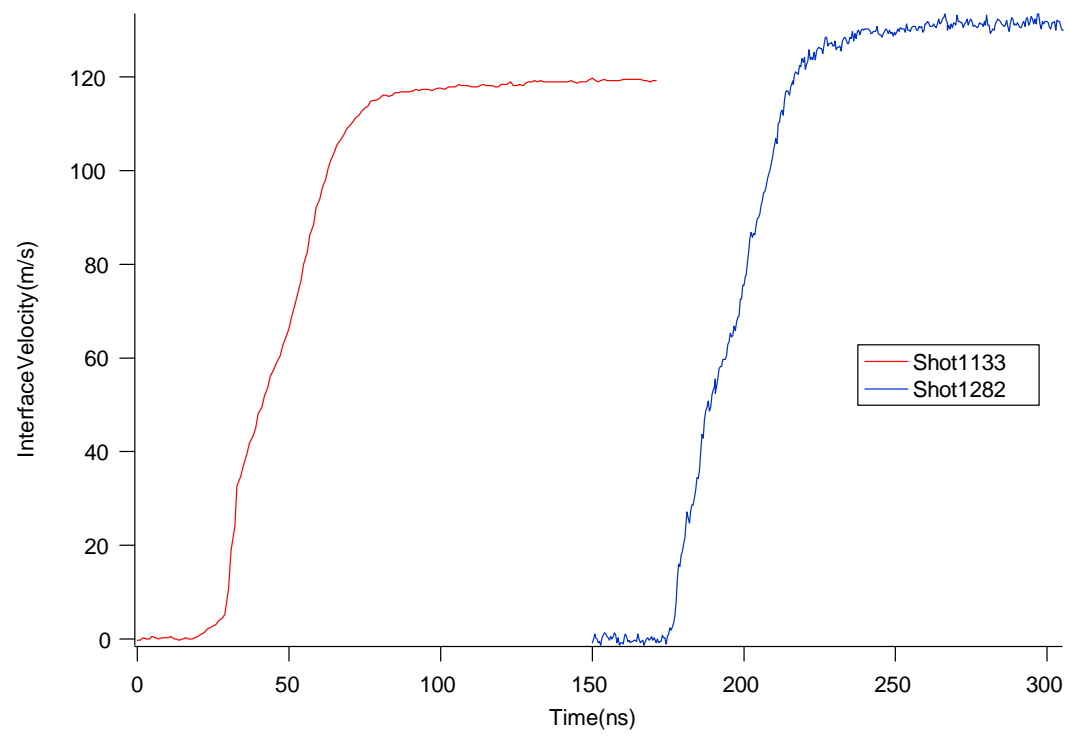

Figure 2. Wave profiles generated by the two successful experiments performed on [001] Ta single crystals. The minimal amount of elastic precursor advance from the plastic wave is due to the relatively thin dimension of the targets (about $500 \mu \mathrm{m}$ ). 


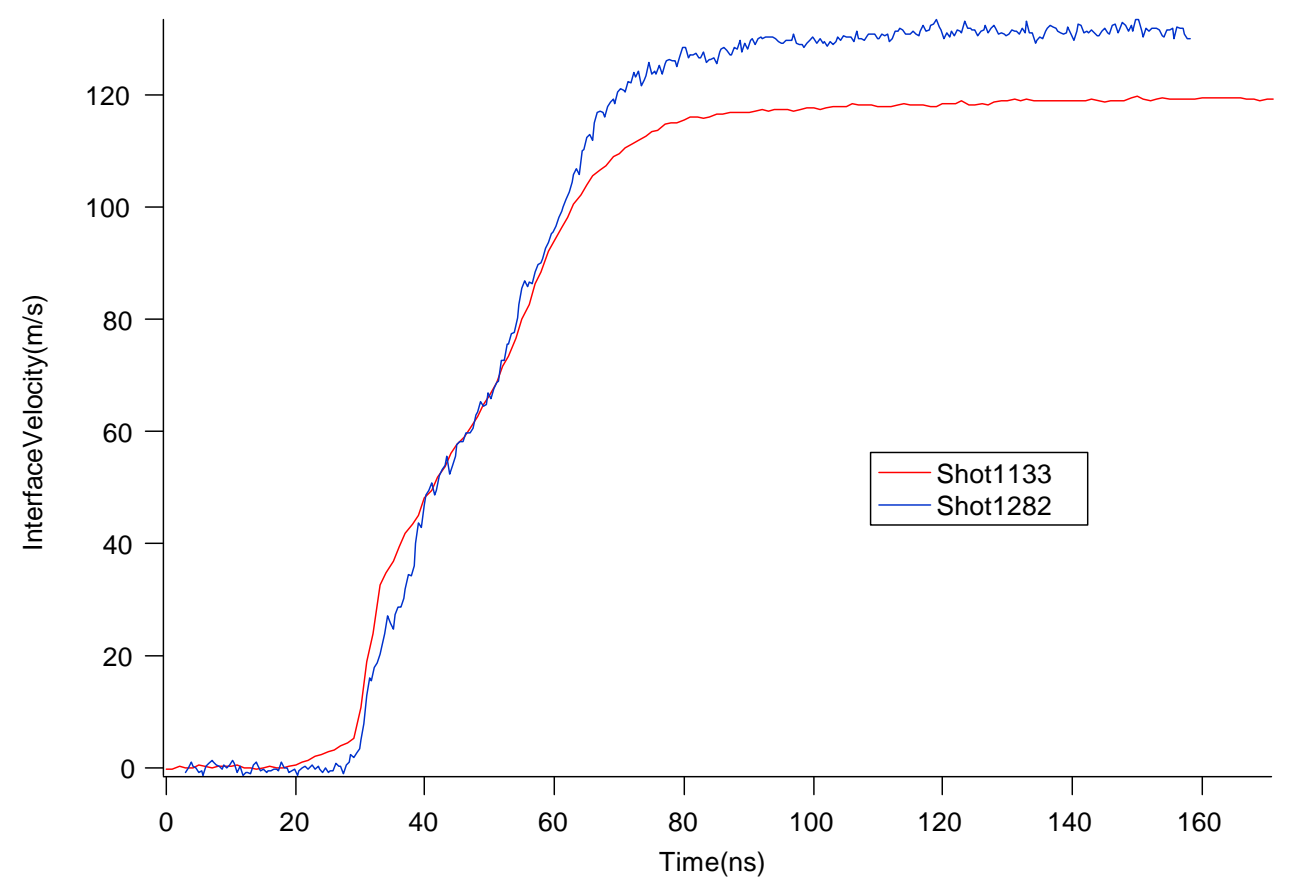

Figure 3. Overlay of the two wave profiles shown in figure 2. The agreement is good in terms of the overall structure of the wave. Note that shot 1282 was at a slightly higher impactor velocity, and hence the peak interface velocity (and pressure) was higher.

The wave profiles can be used to determine the HEL and the "plastic strain rate" during shock loading:

\section{Shot 1133: $\mathrm{HEL}=1.84 \mathrm{GPa}$, plastic strain rate $=6.4$ e 5}

\section{Shot 1282: $\mathrm{HEL}=0.89 \mathrm{GPa}$, plastic strain rate $=7.3$ e 5}

The authors believe that the HEL value of $0.89 \mathrm{GPa}$ for shot is unusually low for bcc Ta, and in conjunction with this the wave profile for this shot appears to have an anomalous break suggesting 2 HEL values, which is not observed in Ta. Therefore we feel the HEL reported for shot 1282 needs to be discounted, however we are not sure what the origin of the discrepancy is. The strain rates calculated for both wave profiles are in the expected range of between $10^{5}$ to $10^{6} \mathrm{~s}^{-1}$.

\subsection{Shock Recovery}

We expect the dislocation substructure and other lattice defect generation at very high strain rate to be quite different than those at lower strain rate due to phonon drag and nonconservative motion. To study the dislocation structures of materials subjected to weakshock, we will perform gas gun experiments similar to those described above, but with 
the intent of recovering the materials after the shock wave has traversed the orientated single crystal.

The shock recover system uses a set of momentum-trapping rings to eliminate lateral deformation of samples during planar impact as shown in figure 3. After the flyer plate impact the sample is then propelled down-range into a soft catch device filled with wool and water, as shown in figure 4. In previous applications, polycrystalline samples have been recovered with little or no secondary deformation. For our single crystal experiments we surrounded disks of single crystal Ta with rings of poly-crystalline Ta. Two shock recovery experiments have been performed and TEM analyses of the recovered samples is in progress.

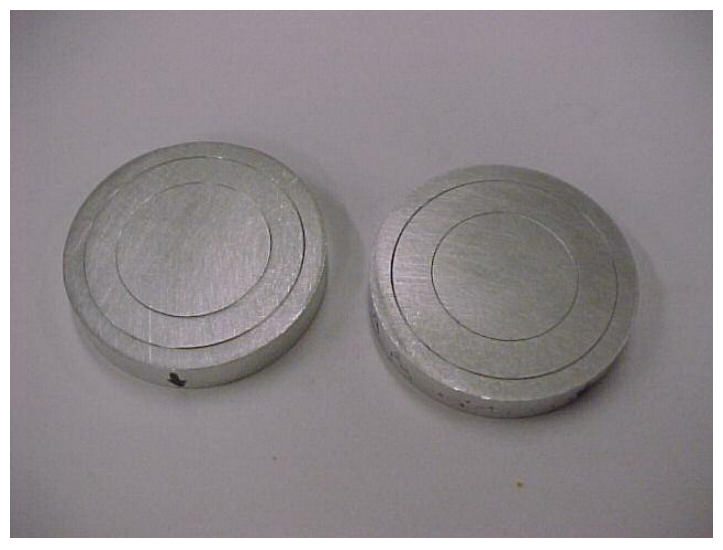

Figure 3. Two Ta shock recover assemblies. The inner disk shown in the photograph is a low-quality $\mathrm{Ta}$ [001] single crystal, in which the high-purity Ta [001] single crystal test sample was inserted with great precision to minimize "free-surfaces" or gaps that can cause wave reflections.

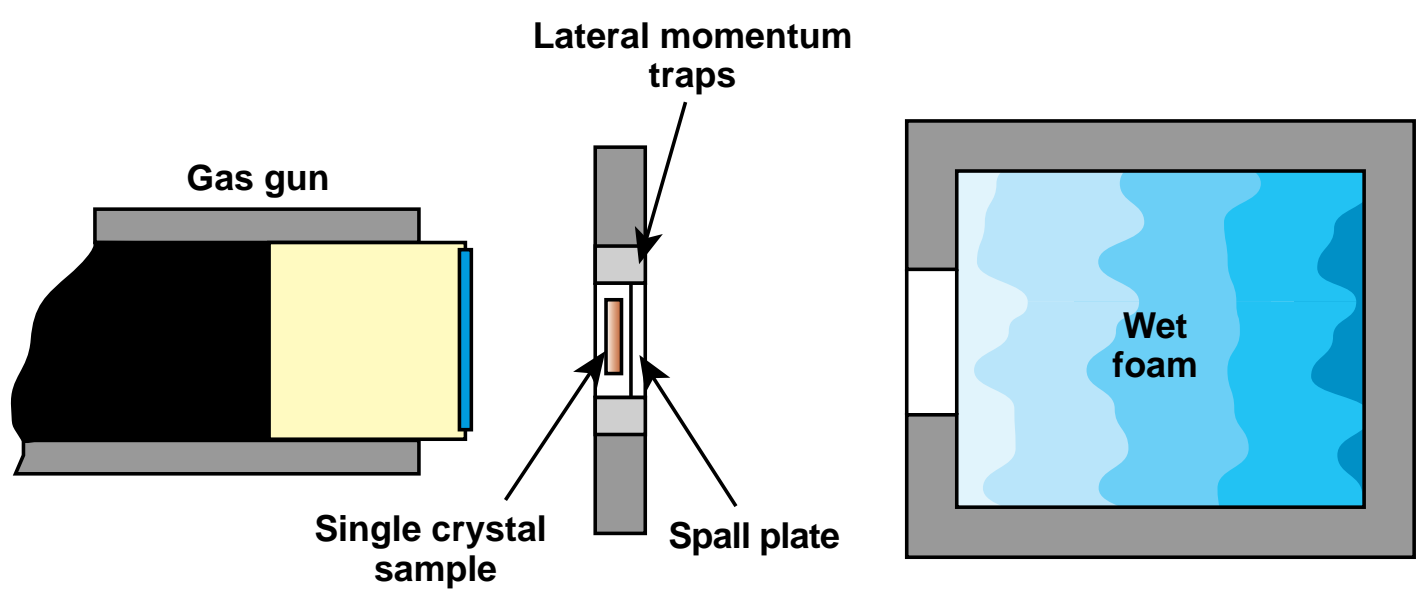

Figure 4. A "soft" recovery tank was designed and constructed to strip the lateral momentum traps and catch the single crystal sample in its anvil. Two successful experiments were performed. 


\section{DD Simulations of Wave Profiles}

The development of DD simulations capabilities to model the passage of shocks has matured significantly over the past few year, particularly due to the work of $\mathrm{H}$. Zbib and co-workers at Washington State University. Although there work up to this time has been mainly on modeling of strong shocks traversing fcc metals $(\mathrm{Cu})$, the work clearly demonstrates the feasibility of validation of key dislocation generation and mobility physics issues in the "weak-shock" regime where phonon drag is expected to be dominate.

\subsection{Dislocation Dynamic Plasticity Model and Simulation}

Multiscale dislocation dynamic plasticity model (MDDP) developed at Washington state university is used to examine the shock wave structure and plastic deformation under shock loading. The MDDP model is based on fundamental physical laws that govern dislocations motion and their interactions with various defects and interfaces. The model merges two scales, the nano-microscale where plasticity is determined by explicit three dimensional dislocation dynamics analysis providing the material length scale, and the continuum scale where energy transport is based on basic continuum mechanics laws. The detail of the framework and basic equations can be found in numerous articles provided by Zbib and co-workers. The model is based on the basic laws of continuum mechanics, i.e. linear momentum balance and energy balance:

$$
\begin{aligned}
& \operatorname{div} S=\rho \dot{v} \\
& \rho C_{v} \dot{T}=K \nabla^{2} T+S \cdot \dot{\varepsilon}^{p}
\end{aligned}
$$

where $v=\dot{u}$ is the particle velocity,,$C_{v}$ and $K$ are the displacement vector field, mass density, specific heat and thermal conductivity. Then, the strain rate tensor $\dot{\varepsilon}$ is decomposed into an elastic part $\dot{\varepsilon}^{e}$ and plastic part $\dot{\varepsilon}^{p}$ which when combined with the classical Hooke's law yields:

$$
\stackrel{\circ}{\mathrm{S}}=\left[\mathrm{C}^{\mathrm{e}}\right]\left[\dot{\varepsilon}-\dot{\varepsilon}^{\mathrm{p}}\right] \dot{\varepsilon}^{\mathrm{e}}, \stackrel{\circ}{S}=\dot{S}-\omega S+S \omega, \omega=W-W^{p}
$$

$C^{e}$ is, in general, the anisotropic elastic stiffness tensor for cubic symmetry, . is the spin of the microstructure and it is given as the difference between the material spin $W$ and plastic spin $W^{p}$. The evaluation of the plastic strain increment is performed in the discrete dislocation dynamics component of the model, involving massive computations of dislocation-dislocation interaction, motion, multiplication, annihilation, etc. The reader is referred to various papers dedicated to the development of this model (Hirth et al., 1998, Zbib et al, 1998-2002, Rhee et al, 1998). The resulting system of equation is solved using that standard finite element method. 
$M D D P$ simulations are performed to investigate the deformation process at high strain rates in copper single crystals. The simulations are designed to mimic uniaxial strain loading at extreme conditions of high strain rates ranging between $>10^{6} / \mathrm{s}$, and short pulse durations of few nanoseconds (Loveridge et al. 2001). As illustrated in figure 5, the simulation setup consists of a block with dimensions $2.5 \quad \mathrm{~m} \times 2.5 \quad \mathrm{~m} \times 25 \quad \mathrm{~m}$. In order to achieve the uniaxial strain involved in shock loading, the four sides are confined so that they can move only in the loading direction. The bottom surface is rigidly fixed. To generate the stress wave a velocity-controlled boundary condition $\left(v_{p}\right)$ with finite rise time $\left(t_{\text {rise }}\right)$ is applied on the upper surface over a short period of time $\left(t^{*}\right)$. In this case, $v_{p}$ corresponds to the average strain rate and $t^{*}$ is the pulse duration. The upper surface is then released and the simulations continue for the elastic wave to interact with the existing dislocations.

The loading and the boundary conditions are summarized in the following equations.

$$
\begin{array}{lr}
u_{z}(t)=-v_{p} t^{2}, & 0 \leq t \leq t_{\text {rise }}, \quad \text { at } z=\frac{L_{z}}{2} \\
u_{z}(t)=-v_{p} t, & t_{\text {rise }} \leq t \leq t^{*}, \quad \text { at } z=\frac{L_{z}}{2} \\
u_{z}(t)=0 & \text { at } z=-\frac{L_{z}}{2} \\
u_{x}(t)=0, & \text { at } \quad x=-\frac{L_{x}}{2}, \frac{L_{x}}{2} \\
u_{y}(t)=0, \text { at } & y=-\frac{L_{y}}{2}, \frac{L_{y}}{2}
\end{array}
$$

Where $L_{x}, L_{y}$, and $L_{z}$ are the lengths of the computational cell in the $\mathrm{x}, \mathrm{y}$ and $\mathrm{z}$ directions respectively, $u_{x}, u_{y}$, and $u_{z}$ are the displacement components.

Frank Read loops distributed on different slip planes are used as agents for dislocation generation. For copper, the length of each source is $0.70 \mathrm{~m}$.

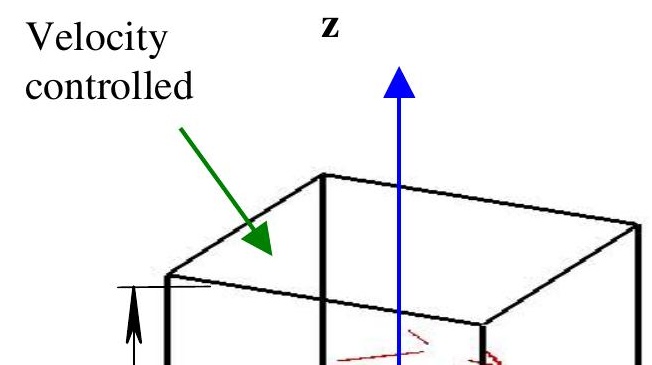


Figure 5. Setup of the simulation cell.

Figure 6 shows stress snapshots in the loading direction ( 33 ) of a wave propagating in copper single crystal shocked to a peak stress of $5.0 \mathrm{GPa}$ for 1.50 nanoseconds pulse with $0.50 \mathrm{~ns}$ rise time. The wave profile consists of three distinct regions: 1- wave front in which the stress increases from zero to the peak value over a period equivalent to the rise time, 2) plateau of the peak stress which stays for a period equivalent to the holding time of the peak stress, 3) and a release part where the peak stress decreases from its peak decrease almost instantaneously from the peak value to zero. The tail of the release wave shows fluctuations that are attributed to the FE mesh. The frequency of these fluctuations is proportional to the FE density (Simirnova et al 2000, Shehadeh et al in preparation). The wave profile consists of 1) a wave front that increases from zero to the peak value of stress 2) fluctuations about the peak stress for a period of time corresponds to the pulse duration and 3) a release part where the stress decreases from its peak value to zero. As the wave propagates the pressure wave the width of the plateau decreases till it vanishes after which the attenuation of the wave takes place and the release wave becomes broader. The attenuation in the pressure is due to the faster moving release wave compared to the wave front and also to the defect generation process, which dissipates part of the wave energy into heat to the process of dislocation motion and multiplication. 


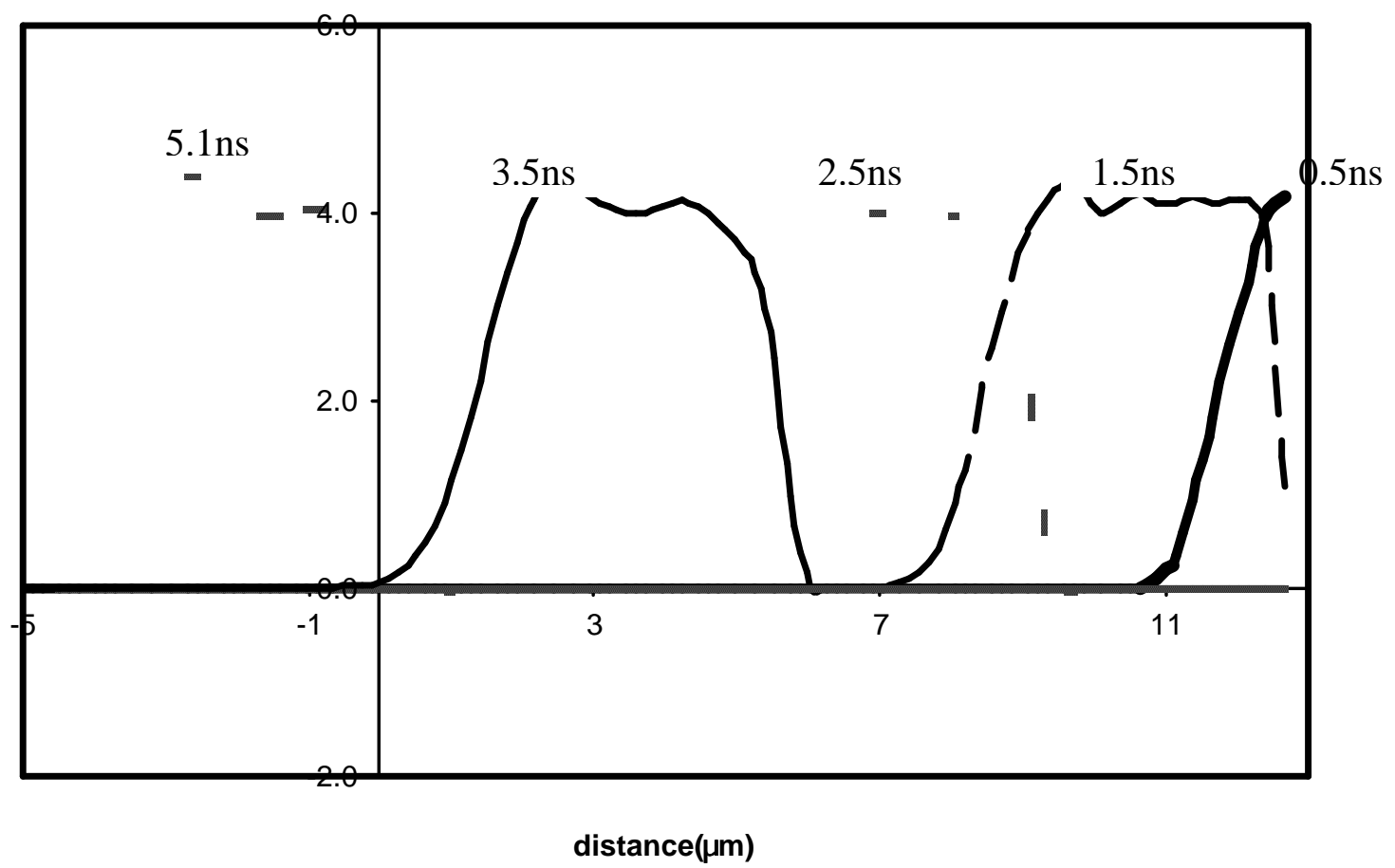

Figure 6. Snapshots of a wave propagating in copper single crystal shocked to 4.5 GPa peak pressure for 1.50 nanoseconds pulse duration.

\subsection{Evolution of Dislocation Structure (Simulation Results)}

The dislocation microstructure generated by the shock waves depends on a number of shock wave and material parameters (Meyers 1994). Among shock wave parameters, pressure (strain rate) is the most important. Murr and Wilsdorf (1978) observed that the dislocation density varies as a square root of the applied pressure. Shock pulse duration is another important shock wave parameter in controlling the microstructure of the dislocations. Pulse duration is related to the time required for the dislocations to reorganize. For the case of dislocation cell structure, it was observed that as the pulse duration increases, the cell walls become better defined (Wright et al. 1981). In principal, it should be possible to observe all of these effect in the DD simulation capability describe herein if dislocation evolution is by dislocation multiplication (e.g., Frank-Read sources).

The effect of the shock (about $4.5 \mathrm{GPa}$ ) passage through an initial dislocation structure of Frank-Read sources (shown in Figure 5) is a dramatic increase in dislocation density, as 
shown in Figure 7. Bands of dislocation structure probably associated with the $\{111\}$ slip planes are evident. It is worthy to mention that multiple deformation bands were formed suggesting that multiple slip planes were activated due to crystal orientation effect. Clearly a strong connections between the DD simulation results and material from shock recovery experiments examined by TEM are feasible.

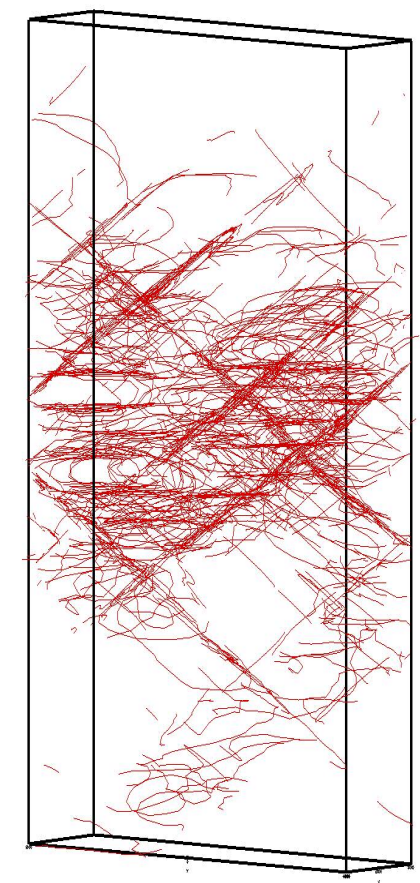

Figure 7. The dislocation microstructure in copper crystal at $4.5 \mathrm{GPa}$ peak pressure and $1.50 \mathrm{~ns}$ pulse duration for crystal oriented in the [001] orientation

\subsection{Summary and Conclusions}

The task of generating experimental and simulation "data" for weak-shocks that can be compared one-to-one is difficult at best. However, the data presented in this report clearly shows the feasibility of such an effort, and that if it is accomplished, significant progress towards the validation of dislocation multiplication and mobility physics issues can be made.

From the experimental standpoint, it has proven to be very difficult to obtain wave profiles on very thin targets $(\sim 500 \mu \mathrm{m})$. Additional attempts at generation of weak-shock wave-profiles would clearly be advantageous. Also, while the shock recovery efforts were successful, we still need to perform TEM analyses of the recovered single crystal, which involves a very difficult thinning operation to observe dislocation structures in the area of zero to $500 \mu \mathrm{m}$ from the impact surface. 
In the area of simulations, it appears that most, if not all of the DD simulation capability to model the experiments is in place (with the proviso that dislocation generation does not occur via spontaneous nucleation which would require atomistic simulation.) The challenges will mainly be in the areas of computational capability, i.e., can a large enough volume of material be simulated so that the entire length of the experimental test sample can be simulated $(500 \mu \mathrm{m})$, and at the same time will the cross sectional area is sufficient to capture realistic dislocation structure evolution, for example dislocation cell structure. Given the availability of time on parallel computers, and massively parallel DD simulation codes, the simulation of the experimentally observed wave profiles are clearly within reach.

\section{References}

Steinberg, D. J., S. G. Lund (1989), J. Appl. Phys. (1989) 65, 1528 